Janja Polajnar*

Universität Ljubljana
UDK 811.112.2'42:004.773

DOI: 10.4312/linguistica.59.1.267-285

\title{
ZUR INTERTEXTUALITÄT IN POSTINGS: \\ EINE EXEMPLARISCHE ANALYSE DEUTSCHSPRACHIGER POSTINGS IN KOMMENTARFOREN ZU ONLINE-ZEITUNGSARTIKELN ZUM BOLOGNA-PROZESS
}

\section{PRÄMISSEN}

Anhand der zunehmenden Internetnutzung finden sich viele traditionelle Textsorten, hier kommunikative Praktiken ${ }^{1}$ genannt, heutzutage auch in den digitalen Medien wieder: Für die hier anvisierten Postings ${ }^{2}$ der Kommentarforen, die mehr oder weniger moderiert im Anschluss an einen Online-Artikel erscheinen, stellt Weidacher (2017) fest, dass diese als textuelle Praktiken an Leserbriefe in den klassischen Printmedien anschließen. Ihre Mediengebundenheit ist allerdings so zentral, dass hier von einer neuen kommunikativen Praktik ausgegangen werden kann (vgl. Weidacher 2017, Spieß 2018). Postings zeichnen sich nämlich durch „die Möglichkeit einer dialogischen Orientierung [aus], die Auswirkungen auf die Form, die Funktionalität und die Semantizität der Praxis der User*innen-Kommentare hat" (Spieß 2018: 159). Wie Leserbriefe beziehen sich auch Postings auf die Artikel von Online-Zeitungen, zeichnen sich außerdem durch „das hohe Interaktionspotenzial“" (Zimmermann 2017: 226) bzw. durch interaktive Reaktionsmöglichkeiten auf Postings anderer UserInnen aus. Zwar ist Interaktivität auch für Leserbriefe nicht unmöglich, stellt jedoch eher die Ausnahme dar (vgl. ebd.).

Anhand der skizzierten Bezugnahmen wird in Anlehnung an Fandrych/Thurmair (2011: 114) bei Intertextualität von einem für Postings konstitutiven Prinzip ausgegangen, denn Postings beziehen sich inhaltlich und intertextuell systematisch aufeinander. Die Beschreibung vielfältiger intertextueller Bezüge in Kommentarforen stellt insofern

\footnotetext{
janja.polajnar@ff.uni-lj.si

1 „Praktiken” bzw. „Praxen” haben sich in den letzten Jahren nicht nur in der Linguistik zu einem Grundbegriff entwickelt, mit dem „Sprache, Text, Verhalten und Kommunikation neu” gedacht werden, indem „Prozessualität, Materialität, Verkörperung und soziale Routinen [...] ins Zentrum des Gegenstandsverständnisses [rücken]" (Deppermann/Feilke/Linke 2016: 1). Ausgehend vom Bourdieus Praktikenansatz (2015) erarbeiteten Deppermann/Feilke/Linke (2016) aus der linguistischen Perspektive verschiedene Bestimmungskategorien für die Beschreibung von kommunikativen Praktiken. Postings als kommunikative Praktik werden nach diesen Bestimmungskategorien überzeugend von Spieß (2018) beschrieben. Im vorliegenden Beitrag sind unter 2. die wichtigsten Charakteristika zusammengefasst.

2 In der (medien-)linguistischen Literatur werden Postings unterschiedlich bezeichnet: (gepostete) Kommentare/ Kommentare zu Posts (Große 2015), User*innen-Kommentare in Foren (Spieß 2018), Leser-Kommentare (Zimmermann 2017) u. a.
} 
eine Herausforderung dar, als durch die starke mediale Prägung sich auch die intertextuellen Bezüge veränder(te)n. Hierbei soll im vorliegenden Beitrag die Frage fokussiert werden, welchen Einfluss mediale Prägung auf die intertextuellen Bezüge als Resultat veränderter Textgestaltung hat. Dies soll anhand der Analyse und Beschreibung intertextueller Bezüge in Postings dreier Kommentarforen im Analyseteil geleistet werden. Davor sollen in einem theoretischen Abriss zunächst die Charakteristika von Postings als junger sprachlich-kommunikativer Praktik sowie im Hinblick auf ihre Texthaftigkeit und dann die Intertextualität erläutert werden.

\section{POSTINGS IN KOMMENTARFOREN: EINE JUNGE SPRACHLICH- KOMMUNIKATIVE PRAKTIK}

Das Medium Internet ermöglicht, dass man eine individuelle, zumeist kritische Meinungsäußerung zu Artikeln in Online-Zeitungen oder anderen Postings öffentlich in Form von kurzen anonymen Kommentaren bzw. Postings wie in Echtzeit äußern kann. Weidacher geht ,im Falle von Postings in einem Kommentarforum von extern dialogisch verknüpften Texten [aus] [...], die zugleich Teiltexte eines intern dialogisch strukturierten komplexen Textes, des Forums nämlich, sind“" (Weidacher 2017: 163). Wie bei anderen Hypertexten bzw. -media stellt sich also auch bei Postings in einem Kommentarforum die Frage nach der Abgeschlossenheit bzw. Grenzbestimmung von Texten. Hierbei wird mit dem Begriff "Teiltexte“ in der obigen Definition meines Erachtens nur auf die hierarchischen Verhältnisse der Texte zueinander verwiesen und nicht die Frage der Texthaftigkeit angeschnitten; im vorliegenden Beitrag werden in Anlehnung an Auerbach-Kutscher (2018: 76-81) sowohl einzelne Postings als auch der Hypertext (Kommentarforum) selbst als Texte aufgefasst, denn auf beiden Ebenen kann von einer kohärenten und funktionalen Größe ausgegangen werden.

Zimmermann (2017: 320) stellt für Postings fest, dass es den Verfassern von Postings inhaltlich bzw. argumentativ nicht um die ,gemeinschaftliche Ausdifferenzierung einer rationalen öffentlichen Meinung" geht, sondern darum, eigene Ideen, Befindlichkeiten und eine Grundstimmung zu kommunizieren (vgl. Große 2015: 62); auch ist eine inhaltliche Kontinuität des Zeitungsartikels als Referenztextes nur teilweise gegeben (vgl. Große 2016: 61). Im Vergleich zu Leserbriefen ${ }^{3}$ stellen Postings ein direktes, schnelles und authentischeres öffentliches Kommunikationsinstrument sowie „ein leicht zugängliches und wenig beschränktes Forum für textbasierte, asynchrone diskursive Partizipation dar", was in hohem Beteiligungsniveau resultiert (Zimmermann 2017: 117); Online-Artikel zu brisanten Themen können so in relativ kurzer Zeit von einigen Stunden mehrere Hundert Postings erhalten (ebd.). Wie dialogisch orientiert Postings in Kommentarforen schlussendlich sind, hängt zum einen vom Online-Medium und zum anderen von der Aktivität der UserInnen ab (vgl. Spieß 2018: 159). Die hier analysierten Kommentaroren weisen trotz gleichen Themas und Mediums (Zeit-Online) sehr unterschiedliche dialogische Orientierung auf.

3 Weitere Ähnlichkeiten und Unterschiede von Leserbriefen und Postings sind der Dissertation zu deliberativer Partizipation im Bereich der Politikwissenschaft von Tobias Zimmermann (2017) zu entnehmen. 
Postings in Kommentarforen werden aufgrund ihrer medialen Prägung, die die jeweilige Praktik in ihrer Form, Funktionalität und Semantizität beeinflusst, als eine neue reaktive sprachlich-kommunikative Praktik aufgefasst (vgl. Weidacher 2017: 154; Spieß 2018: 159). Da Postings stark an technisch-mediale Voraussetzungen der computervermittelten Kommunikation und die Rahmenbedingungen der jeweiligen Online-Medien angewiesen sind, spricht Androutsopoulos (2016) von „mediatisierten Praktiken“. Neben der für diese kommunikative Praktik zentralen Dialogizität bzw. Interaktion nennt Weidacher (2017: 164-165) für Kommentarforen Hypertextualität, Multimodalität (zumeist durch Verlinkung ermöglicht) sowie Fluidity (bezogen auf das gesamte Forum und nicht auf einzelne Postings) als Charakteristika. Zudem erwähnt er noch die kulturelle Prägung (z. B. Wertmaßstäbe in Bezug auf Höflichkeit oder Tabuisierung von Themen und Ausdrucksweisen) sowie die Prägung durch etablierte Textsorten (z. B. Abschiedsgrüße entliehen aus E-Mails bzw. Briefen). Hierbei schließen Postings als textuelle Praktiken an die kommunikative Praktik der Leserbriefe in den klassischen Printmedien an (vgl. ebd.).

Für die sprachlich-formale Gestaltung der Postings bzw. die Ausdrucksweise der NutzerInnen betrachtet Weidacher zudem die Anonymität sowie den inhärenten Zwang zur Kürze als grundlegend. Vor allem das anonyme Kommunizieren scheint dazu beizutragen, dass sich die NutzerInnen insbesondere bei brisanten Themen „direkter, unhöflicher, z.T. unflätig ausdrücken (,flaming')“ (Weidacher 2017: 164; vgl. Fandrych/ Thurmair 2011) sowie zum ironischen und sarkastischen Formulieren neigen. Aufgrund der Echtzeitschriftlichkeit kann es zu grammatischen und orthographischen Fehlern sowie zu emulierter Mündlichkeit kommen, wobei letztere hinsichtlich des interaktionalen dialogischen Charakters der Kommunikation stellenweise adäquat erscheint (ebd.). Große (2015: 57) hingegen stellt für Kommentarforen keine eindeutig nähe- bzw. distanzsprachliche Orientierung fest, denn manche Postings erinnern an Nachrichtentexte und manche an Gesprächssequenzen. Die Autorin verweist auch auf ihre Heterogenität, die Schlussfolgerungen zu sprachlich generischen oder konstitutiven Spezifika kaum möglich macht, stellt jedoch ihre Nähe zu Chat-Kommunikation und Diskussionsforen fest. Im Hinblick auf Sprachhandlungen überwiegen in dieser meinungsbetonten bzw. kommentierenden sprachlich-kommunikativen Praktik Assertiva, Direktiva und vor allem Expressiva (Weidacher 2017: 164).

Mediengebundenheit nimmt zudem Einfluss auf die strukturellen Rahmenbedingungen von Postings, die sich als sehr heterogen erweisen: Den einzelnen Betriebsmedien stehen nämlich zahlreiche Möglichkeiten zur Verfügung, „das Design, die Funktionen und die Organisation ihrer Leserkommentare zu variieren“" (Zimmermann 2017: 117). In den untersuchten Kommentarforen von Zeit-Online weisen Postings folgende Struktur auf: Username, Nummer des Postings (\#1, \#2 bei den Postings, die sich auf den Referenzartikel beziehen und \#1.1, \#1.2 bei den Postings, die sich auf andere Postings beziehen), Datum und Uhrzeit, Überschrift, Fließtext und bei den Postings, die sich auf andere Postings beziehen, noch der Zusatz: Antwort auf\#1 von (Username) (vgl. Beispiele 10-12).

Online-Artikel und mit ihnen systematisch vernetzte Postings (,Textsorten-in-Vernetzung“ Janich 2008) stellen einen thematisch zusammenhängenden, öffentlichen 
Teildiskurs dar. So wird im vorliegenden Artikel davon ausgegangen, dass sprachliches Handeln in Diskursen und sprachlich-kommunikativen Praktiken verankert ist (vgl. Spieß 2018: 154). Hierbei können Postings den Kommentarforen- bzw. Diskursverlauf wesentlich vorantreiben (vgl. Spieß 2018) und sogar den durch den Online-Artikel gesetzten semantischen Fokus (stark) verschieben. Nach Spieß stellen Postings nämlich „Möglichkeitsbedingungen“ für weitere Postings dar; als sprachliche Praktik bringen sie demzufolge „selbst Kontexte hervor und kontextualisieren sich selbst im Kommunikationsgeschehen“" (Spieß 2018: 159). Schließlich sei noch die spezifische Beteiligungsstruktur der Kommentarforen diskurslinguistisch perspektiviert: Spieß erwähnt neben den UserInnen, die auf den Artikel oder andere Postings reagieren, noch die Medien als ,plurale, kollektive Akteure“; diese ermöglichen nicht nur die Teilnahme am Kommunikationsforum, sondern greifen redaktionell ein und können auf diese Weise „Sachverhalte perspektivieren, tabuisieren, kontrollieren und beschränken“ sowie einen erheblichen Einfluss auf den Diskurs nehmen (Spieß 2018: 159).

\section{INTERTEXTUALITÄT}

Intertextualität bezieht sich im Folgenden ,,auf bewusste, intendierte und markierte Bezüge zwischen einem Text und vorliegenden Texten oder Textgruppen“" (Pfister 1985: 25; vgl. Holthuis 1993: 23 oder Adamzik 2004: 97ff.) und wird aus der Perspektive der Textproduktion als Texteigenschaft aufgefasst. ${ }^{4}$ Hierbei wird durch Referenzsignale und explizite Markierungen in Phänotexten ${ }^{5}$ angezeigt, „welche Referenztexte mit in die transtextuelle Analyse einbezogen werden müssen, um sie (be)deutbar bzw. verstehbar zu machen" (Klug 2018: 115). Der Begriff Text darf sich hierbei nicht nur auf schriftliche und mündliche Einheiten (Brinker/Sager 2006: 7) beschränken, sondern muss auch dialogisch ausgerichtete sowie nichtsprachliche Texte wie Bilder und Musikstücke u. a. (Spieß 2018: 155, Klug 2018: 113-115) mit einbeziehen. Dies sei vor allem dort relevant, wo sich in den sprachlichen Texten Verweisstrukturen auf dialogische Strukturen oder audio-visuelle Elemente eruieren lassen (Klug 2018: 114), was auch für die hier fokussierten Postings von Relevanz ist.

Im Allgemeinen werden intertextuelle Bezüge nach ihrer Art in zwei Globaltypen von Intertextualität unterteilt: Bei der typologischen Intertextualität (Holthuis 1993; Opiłowski 2006) bzw. Systemreferenz (Broich/Pfister 1985) geht es einerseits um Bezugnahmen eines Textes (token) auf ein oder mehrere Textmuster, Textsorten, literarische Gattungen oder Diskurstypen (type). Intertextuell interessant sind hierbei vor allem „Abweichungen von Mustern“ wie Musterbrechung, Mustermetamorphose und Mustermontage (Janich 2019: 192). Andererseits geht es beim hier relevanten zweiten

4 Ein Überblick zu den Intertextualitätsbegriffen siehe Janich (2019: 177-189), Auerbach-Kutscher (2018: 97-137), Opiłowski (2006) u. a.

5 Im Hinblick auf die terminologische Vielfalt in der literatur- und sprachwissenschaftlichen Intertextualitätsforschung seien in Anlehnung an Janich (2019: 178), die terminologisch an sprachwissenschaftliche Zeichentheorie und Textgrammatik anknüpft, Phänotexte diejenigen Texte, die sich auf andere Texte beziehen. Referenztexte sind dagegen Texte, auf die Bezug genommen wird. Analog dazu wird hier von Phäno- und Referenzpostings gesprochen (Janich 2019: 182). 
Globaltyp, der sog. referenziellen Intertextualität (Holthuis 1993) bzw. Einzeltextreferenz (Broich/Pfister 1985) um Bezugnahmen eines konkreten Einzeltextes auf andere Einzeltexte in Form von Zitaten, Anspielungen, Paraphrasen (Holthuis 1993). Breckle/ Enell-Nilsson (2015: 91-92) unterscheiden zudem, ob intertextuell auf einen Text(teil) Bezug genommen wird oder aber der Emittent des Bezugstextes im Hinblick auf seine geäußerte Meinung (sog. Quelle) erwähnt bzw. kommentiert wird. Bei den hier untersuchten Postings, die mit dem Referenzartikel und untereinander systematisch vernetzt sind, ist die Vielfalt an Einzeltextreferenzen besonders hoch und reicht von argumentativen Ergänzungen und inhaltlichen oder metakommunikativen Kommentierungen über Korrekturen bis hin zu Verweisen auf andere beliebige Texte. Hierbei werden die Bezüge zwischen den Postings, denen hier Textcharakter zugesprochen wird, in Anlehnung an Adamzik (2004: 10) zu den intertextuellen Bezügen gezählt.. ${ }^{6}$ Da sich die Intertextualitätsbezüge von Kommunikationsbereich (Domäne) zu Kommunikationsbereich unterscheiden können, sollen sie gegenstandsbezogen erarbeitet werden. Für Werbetexte schlägt Janich (2008: 189-190) beispielsweise folgende Typen vor, die modifiziert auch für die vorliegende exemplarische Untersuchung von Bedeutung sind: (un)markierte Zitate, Anspielungen durch Übernahme von Strukturen, Anspielungen durch Übernahme von zentralen lexikalischen Einheiten, Anspielungen auf den visuellen Code sowie inhaltliche Anspielungen (ohne Übernahme von Strukturen oder zentralen lexikalischen Einheiten). Bei den Typologisierungen sollen nach Pfister (1985) referenzielle Intertextualitätsbezüge nach Intensitätsgrad ausdifferenziert werden (z. B. Grad der Markierung: Zitat vs. Anspielung/Verweis), denn intertextuelle Bezüge weisen einen unterschiedlichen Grad an Explizitheit auf, was mit der Qualität (Typen) und Quantität (Umfang intertextueller Übernahmen, Dichte intertextueller Bezüge sowie Streubreite der Referenztexte) der Intertextualitätssignale zusammenhängt (vgl. Breckle/Enell-Nilsson 2015: 88, 91-92). Gegenstandsbezogen können sich auch die Funktionen unterschiedlicher Formen der Intertextualität voneinander sehr unterscheiden: Während intertextuelle Bezüge in wissenschaftlichen Texten beispielsweise der Nachweispflicht und Anerkennung dienen, erfüllen sie in Zeitungstexten und in der Werbung vorwiegend die aufmerksamkeitserregende Funktion und Lesemotivation (vgl. Janich 2018: 191).

Im Hinblick auf die mediale Prägung und die veränderte textuelle Praktik von Postings wird von einer neuen, medial geprägten intertextuellen Qualität gesprochen (vgl. Heibach 2003, Gray 2006). Diese hängt damit zusammen, dass Texte nicht mehr nur unidirektional aufeinander bezogen sind, sondern ,auf vielfältige Weise wechselseitig miteinander [kommunizieren]" (Berghofer 2009: 71). Gray (2006: 20ff.) behandelt die Intertextualitätsbezüge, die in Kommunikationsforen zwischen Postings intern dialogisch zustande kommen, im Rahmen eines ,voll interaktive[n] Intertextualitätsmodel1[s]". Da die bisherigen Intertextualitätskonzepte und -typologisierungen an den Buchdruck gebunden waren („Medienblindheit der Intertextualitätstheorie“, Heibach 2003:

6 Adamzik (2004: 105) ergänzt die genannten zwei Globaltypen aus textlinguistischer Perspektive um a) intertextuelle Beziehungen zwischen Teiltexten eines Textes und b) intertextuelle Beziehungen zwischen Textsorten. 
98-99), soll in der exemplarischen Analyse erprobt werden, ob sich mit herkömmlichen Intertextualitätstypen auch intertextuelle Strategien in Postings der Kommentarforen adäquat erfassen lassen.

Intertextuelle Bezugnahmen sind zudem für Diskurse konstitutiv und werden als „das offensichtlichste Kennzeichen der diskursiven Verfasstheit von Sprache" verstanden (Spitzmüller/Warnke 2011: 189; Hervorhebung im Original). Aus diesem Grund wurden in dieser exemplarischen Untersuchung Online-Artikel samt Kommentarforen zum hochschulpolitischen Thema der Bologna-Reform berücksichtigt.

\section{EXEMPLARISCHE ANALYSE: NETZE INTERTEXTUELLER BEZÜGE IN POSTINGS DER KOMMENTARFOREN}

Anhand der exemplarischen Analyse von 119 Postings in drei Kommentarforen (KF) von Zeit-Online, die diskursiv an die hochschulpolitische Debatte der Bologna-Reform anknüpfen, sollen zentrale Aspekte intertextueller Bezüge und deren Funktionen von Postings der Kommentarforen gegenstandsbezogen nach folgenden Aspekten differenziert beschrieben werden: Erstens müssen intertextuelle Verweise und deren Funktionen nach Typen von Referenztexten (Online-Artikel, Postings, beliebiger Text) getrennt analysiert und beschrieben werden. Zweitens soll die Quantität intertextueller Bezüge berücksichtigt werden, indem zum einen der Umfang intertextueller Übernahmen (Text, Absatz, Satz(teil), Wort(gruppe)) und zum anderen die Dichte intertextueller Bezüge sowie die Streubreite der Referenztexte berücksichtigt werden. Drittens soll überprüft werden, ob Übernahmen wegen ihres Umfangs oder ihrer Platzierung nicht die Struktur von Postings beeinflussen (Strukturalität).

Für die Beschreibung unterschiedlicher Formen der Intertextualität in Postings wird Janichs strukturell ausgerichtete Typologisierung von Einzeltextreferenzen in Werbetexten (2008: 189-190) modifiziert herangezogen. Ihre Klassifikation schließt zum einen an Karrer (1985: 98-100) an, der unterscheidet, ob die Struktur, lexikalische Elemente oder Struktur und lexikalische Elemente vollständig oder unvollständig übernommen werden sowie ob Reproduktion oder Anspielung vorliegen. Zudem wird in Janichs Typologisierung Burgers Klassifikation zur Modifikation von Phraseologismen (Burger et al. 1982: 68-102) berücksichtigt.

\subsection{Formen der Intertextualität}

Gegenstandsbezogen sollen bei der Postingsanalyse aufgrund technisch-medialer Eigenschaften neben Bezügen auf Text(teil)e auch Bezugnahmen auf auditive und visuelle Elemente, sei es in Form von Zitaten (Bild-, Musikzitate) oder Anspielungen, berücksichtigt werden. Hierbei sind folgende Formen der Intertextualität denkbar:

- markierte und unmarkierte Zitate (Text-, Bild-, Musikzitate),

- Übernahme von (syntaktischen) Strukturen bei lexikalischer Modifikation,

- Verwendung von zentralen lexikalischen Elementen bei struktureller Modifikation,

- Übernahme von zentralen lexikalischen Elementen ohne offensichtliche Orientierung an syntaktischer Struktur, 
- inhaltliche Anspielung ohne Übernahme von lexikalischen Elementen oder Strukturen (Anspielung auf die Struktur),

- Anspielung auf den audio-visuellen Code.

$\mathrm{Da}$ im Analysekorpus bei einigen Formen keine Belege ermittelt werden konnten, werden im Anschluss nur die häufigsten Formen (fett) anhand von Beispielen veranschaulicht.

\subsubsection{Markierte und unmarkierte Zitate}

Unter Zitaten sind vollständige, syntaktisch und lexikalisch identische Übernahmen von Referenztext(teilen) zu verstehen, die im Phänotext entweder markiert (meist durch Anführungszeichen) oder unmarkiert vorkommen. In Postings ist es technisch-medial zudem möglich, dass auch Bilder oder Musikstücke zitiert werden (Bild-, Musikzitate), was im Analysekorpus allerdings nicht der Fall ist.

In den analysierten Postings werden häufig Zitate aus den Referenzpostings, seltener aus Zeitungsartikeln übernommen (insgesamt $40 \%$, vgl. Tab. 1): Erstens fällt im Hinblick auf Zitate auf, dass die Referenztextstellen der vorangehenden Postings nicht wie oft in den Leserbriefen paraphrasiert werden, sondern häufig längere Zitate (Sätze oder sogar Absätze) durch Anführungszeichen markiert und vorangestellt bzw. abgesetzt übernommen werden. Dies sei auf das einfache Kopieren von Referenztextteilen, vor allem aber auf die Explizierung der Referenztextteile in der Flut unterschiedlicher Postings zurückgeführt. Letzteres unterstützt insofern den Leserfluss, als dadurch dem Leser explizit gemacht wird, welche Textstelle aus welchem Posting nun kommentiert wird. Durch das Einbinden von umfangreichen Zitaten wird am Textanfang bzw. an exponierten Stellen von Postings der Intertextualitätsgrad erhöht, denn dadurch wird visuell stark die Struktur der Phänopostings beeinflusst. So wird im Beispiel (1) ein Satz aus dem vorangehenden Posting zitiert, um im Anschluss kritisch kommentiert zu werden.

Zweitens werden neben längeren Zitaten in Postings oft Wortgruppen bzw. Satzteile zitiert, die man explizit kritisch kommentieren oder, wie im Beispiel 2, korrigieren möchte. Obwohl in den Postings in erster Linie Inhalte diskutiert, kommentiert und ergänzt werden, wie etwa, ob die Bologna-Reform etwas mit der EU zu tun hat (Beispiel 7), oder ob die Aufnahmekriterien mit der Einführung von Bacherlor und Master zusammenhängen (Beispiel 2), liegen auch Postings vor, in denen die falsche Rechtschreibung und/oder Bedeutung verwendeter Wörter oder Wortgruppen implizit und/ oder explizit thematisiert werden. In den Beispielen 1 und 2 erfolgt dies anhand der im Ausgangsposting, zitiert im Beispiel 1, falsch geschriebenen und verwendeten Wortgruppe bremer Fachabitur. Während im Beispiel 1 eine implizite Korrektur im Postingtext vorliegt (Bremer (Fach-)Abiturienten), wird im Beispiel (2) explizit sowohl auf die falsche Rechtschreibung der Wortgruppe mit einer im akademischen Schreiben geläufigen Fehlerkennzeichnung sic! (,,bremer [sic!] Fachabi“) als auch auf ihre bedingte semantische Adäquatheit verwiesen (Beispiel 2). 
(1) \#1.2 - 23. April 2015, 20:14 Uhr 8

Norddeutschen-Bashing

„Erst hat man die Schleusen geöffnet und jeden auch noch mit bremer Fachabitur studieren lassen"

Was soll eigentlich immer dieses Norddeutschen-Bashing im Zusammenhang mit Bildung? Sollen Ihrer Meinung nach die Bremer (Fach-)Abiturienten überhaupt nicht mehr an Hochschulen zugelassen werden, da die Norddeutschen ja per se zu doof zum Studieren sind? [...]

Antwort auf \#1 von MarsUltor ${ }^{7}$

(2) \#1.3 - 23. April 2015, 13:18 Uhr 1

Alles in den Topf und gut umrühren

Die Zweigliedrigkeit (Bachelor/Master) hat rein gar nichts damit zu tun, wer die Hochschulreife bekommt und wer nicht. [...] Dann muss man auch nicht von einem „bremer [sic!] Fachabi“ sprechen, sondern würde merken, dass diese Leistungsrankings eher den Druck nach oben trieben und gleichzeitig [...] c) ein Fachabi (umgangssprachlich für die Fachhochschulreife) in der Regel nicht zu einem Uni-Studienplatz führt.

Antwort auf \#1 von MarsUltor

Zitate aus Referenzpostings können zudem verwendet werden, um metasprachlich das unangemessene sprachliche Verhalten im Kommentarforum zu kritisieren. Durch den schnellen Posting-Wechsel kann es bei widersprüchlicher Meinung nämlich schnell zu einer direkten und unhöflichen Ausdrucksweise kommen. Im folgenden Postingswechsel wird beispielsweise die unhöfliche Frage aus dem vorangehenden Posting markiert zitiert „können Sie lesen?“, um das sprachliche Verhalten von der-freimaurer euphemistisch als interessanten Diskussionsstil zu bezeichnen. Zudem zitiert MisterMankind die Überschrift von der-freimaurer und beschreibt sie metasprachlich als missverständlich, um die Information in seinem Posting zu begründen.

(3) der-freimaurer

\#8.5 - 23. April 2015, 21:25 Uhr

Können Sie lesen?

Ich habe doch explizit vom Medizinstudium gesprochen und nicht vom

Jurastudium.

(4) MisterMankind

\#8.6 - 23. April 2015, 22:25 Uhr

$\mathrm{Ja} . .$.

Ja, kann ich. Ihre Überschrift lautete dennoch „Staatsexamen - Anwesenheits-

pflicht" und war insofern missverständlich.

7 Postings wurden unverändert bzw. unkorrigiert übernommen, weshalb relativ häufig Rechtschribfehler und sonstige grammatische Fehler vorliegen. 
Übrigens, interessanter Diskussionsstil mit „,können Sie lesen?“،

Antwort auf \#8.5 von der-freimaurer (KF1)

Sonst werden die Überschriften von Referenzpostings zitiert, um den expliziten Besuch auf das genannte Posting herzustellen, wie etwa: Zu Nr. 60 Wessen Absicht? (Posting \#29 von Yeti-1; KF1) oder RE: Wir (Studenten) sind Opfer des Systems (Posting \#11.2 von sake-lees).

Drittens können sich Postings intertextuell auf beliebige weitere Texte beziehen, indem Textteile entweder markiert oder unmarkiert an exponierten oder für die Argumentation wichtigen Stellen zitiert werden oder aber auf ,ausgelagerte“ Texte verlinkt wird. Folgendes Posting ist beispielhaft für die argumentativ-strategische Verwendung eines markierten Zitates aus der Bologna-Erklärung, das die Argumentation, Bologna-Reform sei durchaus mit der EU verbunden, stützt und im Verlauf des Postings durch ein weiteres englischsprachiges Zitat aus der Bologna-Erklärung als Autoritätsbeweis unterstrichen wird.

(5) \#6.2 - 24. April 2015, 23:22 Uhr

nicht harmlos

Die Bologna-Erklärung hat sehr wohl was mit der EU zu tun. Sie wurde von den Bildungsministern der EU Länder in Bologna unterzeichnet und sah eine „Harmonisierung der Architektur der europäischen Hochschulbildung“" vor. [...]

Aus der Bologna Deklaration: ,We need to ensure that the European higher education system acquires a world-wide degree of attraction equal to our extraordinary cultural and scientific traditions." FAIL! (KF1)

Nicht selten konnten an exponierten Stellen von Postings zudem Zitate von Sprichwörtern und weiteren satzwertigen Phraseologismen (vgl. Lüger 1999) gefunden werden, die unterschiedliche Funktionen erfüllen: So wird im Beispiel 2 in der Überschrift eine feste Wortverbindung aus dem Kochdiskurs Alles in den Topf werfen mit einer weiteren gut umrühren kontaminiert, um metaphorisch auf eine unzulässige Verallgemeinerung in der Argumentation des vorangehenden Postings zu verweisen; dies wird im Posting zudem expliziert: Vielleicht etwas mehr informieren und weniger um sich schlagen. Im Beispiel 4 wird anknüpfend an den hochschulpolitischen Diskurs sowohl in der Überschrift als auch am Textende der gereimte politische Spruch der Deutschen Studentenbewegung der 1960er-Jahre zitiert. Mit dem Slogan protestierten Studenten 1967 an der Universität Hamburg gegen fragwürdige Traditionen der Universitätspolitik und forderten deren Demokratisierung sowie die Mitbestimmung der Studentenschaft (vgl. Nath 2007). Hierbei wird bereits durch den unmarkierten ersten Sloganteil in der Überschrift die tradierte Sonderstellung von ProfessorInnen fokussiert, die im Posting nun auf die Anwesenheitspflicht bezogen wird. Am Ende des Postings wird der markierte und als Demo-Spruch gekennzeichnete Slogan stilistisch-effektvoll wiederholt, um die im Posting explizierte Meinung, dass Gesetze für das Fußvolk und nicht für die Professoren seien, pointiert zu resümieren. 
(6) \#8-23. April 2015, 17:46 Uhr 5

Unter den Talaren....

[...] Hier gilt es anzumerken, dass es in Deutschland ebenfalls keine

Anwesenheitspflicht gibt. Auch hier ist der Gesetzgeber eigentlich der Meinung,

die Kompetenz für ein Studium müsse etwas oberhalb des

Sitzfleisches angelegt sein. Es hält sich nur kein Professor bzw. keine

Professorin daran. Gesetze sind etwas fürs Fußvolk, für Professoren in unserem

Lande gelten die nicht. Wie schon die 68-er in ihren

Demo-Sprüche richtig erkannten: „Unter den Talaren - Muff von

1000 Jahren!“‘ (KF1)

Interessanterweise wird in einem der folgenden Postings \#8.12 auf die Ironie der Geschichte verwiesen, denn die 68er von damals sind die (älteren) Profs von heute. Dieses Zitat und das Posting \#8.12 knüpfen an vorangehende Postings desselben Kommentarforums (KF1), in denen die 68er Pädagogen im Zusammenhang mit Aufnahmekriterien thematisiert werden (Beispiel 12), und bilden im untersuchten Diskursfragment einen eigenständigen thematischen Diskursstrang. Diskursiv scheint dem Spruch Unter den Talaren, Muff von 1000 Jahren. eine konstitutive Rolle hinzu, denn er wurde auch im Kommentarforum 2 wiederholt verwendet.

Aufgrund technisch-medialer Eigenschaften computervermittelter Kommunikation werden intertextuelle Verweise auf beliebige Texte bzw. Kommunikate zudem häufig durch Verlinkung hergestellt. Verlinkungen dienen zum einen der Textentlastung, denn das Posting bzw. der Lesefluss wird nicht durch längere Zitate unterbrochen; zum anderen werden interessierte Leser zum Weiterklicken und -lesen animiert. In vielen Postings wird auf den zitierten Link bzw. die zitierte URL durch das kataphorisch verweisende Deiktikon hier verwiesen: Meine Bewerbungserfahrungen [...] können hier nachgelesen werden: http://dtj-online.de/bewe (Posting \#3.1, KF1).

Schließlich sei noch die Nennungen von UserInnen oder AutorInnen bzw. das Zitieren von (User)Namen im folgenden Beispiel genannt, in dem der Verfasser/die Verfasserin samt seiner/ihrer Meinung explizit kritisiert wird.

(1) CarolusVincentius \#6.3 - 25. April 2015, 10:44 Uhr 1

Durch Wiederholung wird's nicht wahr.

Nein, das ist falsch. Nur weil auch EU-Länder dies unterzeichneten, wird es nicht ein „Projekt der EU“. [...] Die Autorin macht es sich nur sehr einfach und behauptet fleißig vieles, was mit Bologna nichts zu tun hat und nur der echauffierte Leser lesen möchte. (Posting \#6.3, KF1)

\subsection{2 Übernahme von syntaktischen Strukturen bei lexikalischer Modifikation}

Intertextuelle Bezüge dieser Art sind im Analysekorpus selten. Folgende Postingüberschrift ist beispielhaft für die Übernahme der syntaktischen Struktur des alten Sprichworts Lehrjahre sind keine Herrenjahre. bei lexikalischer Substitution (Lehrjahre durch Studentenjahre): 
(2) Aquarelle \#8.2 - 23. April 2015, 18:56 Uhr

Studentenjahre sind keine Herrenjahre

Also wenn ich als Dozent regelmäßig Vorlesungen halte, die gerade mal von 1/3 der Studenten besucht werden und am Tag der Prüfung dann die restlichen 2/3 aufschlagen nur um am Ende die Credits einzusacken, würde ich auch eine Anwesenheitspflicht einführen, schlicht, weil es respektlos ist.

\subsection{3 Übernahme von zentralen lexikalischen Elementen ohne offensichtliche Orientierung an syntaktischer Struktur}

In Postings als reaktiven textuellen Praktiken werden am häufigsten (argumentative) Gehalte kommentiert, argumentativ ergänzt, bestritten oder negiert (73\%), wobei formal punktuell die zentralen lexikalischen Elemente aus dem Referenztext (Zeitungsartikel, Postings) wie etwa Bachelor, Uni-Bachelor, Bachelor-Student, Bachelornote oder Master, Masterstudent, Masterabschluss sowie Bologna, Bologna-Reform usw. übernommen werden. Dies soll an einem Beispiel veranschaulicht werden: Im Zeitungsartikel Bachelor: Bologna, Geschichte einer Enttäuschung wird der niedrigste akademische Grad Bachelor und das dahin führende Studium aus der Sicht der Unternehmen wie folgt thematisiert:

(3) [...] Sie wollten unbedingt jüngere Absolventen, nun sind selbst Unternehmen unzufrieden mit dem Bachelor. [...]

[...] Nun beklagen Unternehmen in einer Befragung des Deutschen Industrie- und Handelskammertages (DIHK) die fehlenden Fähigkeiten der Bachelor-Absolventen: Nur 47 Prozent der 2000 befragten Firmen gaben an, dass Berufseinsteiger mit diesem Hochschulabschluss ihre Erwartungen erfüllen. 2007 waren noch 67 Prozent der Firmen zufrieden mit den Bachelor-Absolventen, 2011 waren es bereits nur noch 63 Prozent. [...]

DIHK-Präsident Eric Schweitzer gibt in der Welt zu, dass die Bachelor-Studenten nach zwölf Jahren Schule und dem Wegfall der Bundeswehr doch zu jung sein könnten: „Ein wenig Erfahrung und Horizont schaden sicher nicht.“ Mit den Master-Studenten seien Betriebe hingegen zufrieden. Doch eine Garantie auf diesen Abschluss gibt es für Studenten nicht. (Bachelor: Bologna, Geschichte einer Enttäuschung, 23.4.2015, Zeit-Online)

Darauf wird in zahlreichen Postings (hier nur einige angeführt) intertextuell Bezug genommen, indem die lexikalischen Elemente Bachelor sowie die Bachelor-Absolventen übernommen und argumentativ ergänzt oder kommentiert werden:

(4) MarsUltor \#1 - 23. April 2015, 17:00 Uhr 13

$[\ldots]$

B.A. : Belohnung für Abbruch

$[\ldots]$

Der Bachelor ist ein Versuch zu kaschieren, dass an den Unis Studenten rumlaufen, denen man nie das Abitur hätte geben dürfen. 
(5) Horatio Caine \#2 - 23. April 2015, 17:04 Uhr 39

Was hat man erwartet?

...dass ein Bachelor Student genauso viel kann wie ein ,alter“ Diploms- oder Examensstudent oder ein Masterstudent, ich als Unternehmer aber nicht mehr so viel für den bezahlen muss?

(6) BergFrank \#5 - 23. April 2015, 17:35 Uhr 28

Der Bachelor war auch die fixe Idee, einfach an billige Absolventen mit geringen Einstiegsgehältern zu kommen. Einen Akademiker zum Preis eines Facharbeiters mit Fortbildung.

\subsubsection{Inhaltliche Anspielung ohne Übernahme von lexikalischen Elementen oder Strukturen}

Nicht selten wird der (argumentative) Gehalt des Online-Artikels, seltener eines Referenzposting kommentiert, argumentativ ergänzt oder bestritten, ohne im Posting lexikalische Einheiten und Strukturen aus dem Referenztext zu übernehmen (13\%). Folgender Artikelausschnitt aus Zeit-Online und einem Posting sind beispielhaft für eine inhaltliche Anspielung: Im Zeitungsartikel (Beispiel 12) wird eines der für die Unternehmen zentralen Ziele der Bologna-Reform, verkürzte Studienzeiten, thematisiert, was jüngere Absolventen zur Folge haben soll. Die Bachelor-Absolventen sind nach der Reform zwar jünger, aber die Unternehmen beklagen sich nun über ihre fehlenden Fähigkeiten. Im anschließenden Posting (Beispiel 10) nimmt der User intertextuell auf die verkürzten Studienzeiten (in $3 \mathrm{Jah}$ ren im Eilturbo den Stoff durchgeprügelt) und jüngere Absolventen (sind gerade einmal 19, wenn sie den Abschluss haben) Bezug, um kritisch auf die unrealistischen Erwartungen der Unternehmen zu verweisen, dass man mit 19 zwar eine Tätigkeit wie etwa Kaffee kochen ausüben kann, die Erwartungen an Akademiker allerdings anders sind.

(7) Die Unternehmen

Vor allem der Wirtschaft lag daran, dass Absolventen jünger werden. Deshalb war eines der Ziele des 1999 eingeführten Bologna-Prozesses, die Studienzeit zu verkürzen - und die Studenten schneller fit zu machen für den Arbeitsmarkt. Nun beklagen Unternehmen in einer Befragung des Deutschen Industrie- und Handelskammertages (DIHK) die fehlenden Fähigkeiten der Bachelor-Absolventen: [...] (Bachelor: Bologna, Geschichte einer Enttäuschung, 23.4.2015, Zeit-Online)

(8) \#2.1 - 23. April 2015, 22:47 Uhr

[...] Ich frage mich auch was die Unternehmen erwarten! Bachelorabsolventen haben in 3 Jahren im Eilturbo den Stoff durchgeprügelt und sind ,im schlimmsten Fall" gerade einmal 19, wenn sie den Abschluss haben. Natürlich kann jeder mit 19 eine Tätigkeit ausüben - aber ich habe das Gefühl, dass Unternehmen von Akademikern doch etwas mehr erwarten als bloß Kaffee kochen! [...]

Antwort auf \#2 von Horatio Caine (KF1) 
Eine inhaltliche Anspielung liegt auch im folgenden Postingwechsel vor, in dem die Aufnahmekriterien im Referenzposting metaphorisch als die Schleusen genannt werden:

(9) \#1-23. April 2015, 17:00 Uhr 13

[...] Erst hat man die Schleusen geöffnet und jeden auch noch mit bremer Fachabitur studieren lassen.

(10) \#1.3 - 23. April 2015, 23:18 Uhr 1

[...] Wenn in den letzten Jahren dennoch etwas an den Aufnahmekriterien geändert wurde, $[\ldots]$

$\mathrm{Zu}$ inhaltlichen Anspielungen, einem formal offenen Typ, können zudem Frage-Antwort-Sequenzen u. Ä. aus nachfolgenden Postings gezählt werden, ${ }^{8}$ die den hohen Interaktionsgrad der Postings veranschaulichen: Folgende Überschriften zweier Postings sind beispielhaft für eine sprachspielerisch-scherzhafte Auseinandersetzung, die durch das Wörtlichnehmen des Phrasems realisiert wird und durch das glücklich augenzwinkernde Emoticon als ironisch gemeint unterstrichen wird:

(11) \#10.1 - 23. April 2015, 18:26 Uhr 1

Vergessen Sie es...9

(12) \#10.2 - 23. April 2015, 18:38 Uhr 1

Ich vergesse nichts ;-) (KF1)

Inhaltliche Anspielungen dieser Art können aber auch unhöflich und beleidigend sein, wie im Postingswechsel in den Beispielen 3 und 4. Vor dem angeführten Postingwechsel bezeugt der-freimaurer hohe Anwesenheitspflicht beim Medizinstudium, wohingegen MisterMankind davon berichtet, dass es im Jurastudium grundsätzlich keine Anwesenheitspflicht gebe. Daraufhin eröffnet der-freimaurer sein Posting (3) mit einer unhöflich angreifenden Frage, einem indirekten Vorwurf, die wie im Face-to-Face-Gespräch vom MisterMankind mit der Affirmationspartikel ja (mit Auslassungspunkten) und anschließend explizit mit Ja, kann ich. beantwortet wird.

\subsection{Häufigkeitsverteilung einzelner Formen der Intertextualität}

Betrachtet man nun die Häufigkeitsverteilung einzelner Formen der Intertextualität nach Referenztexten näher, die die Nutzung dieses Phänomens in einem relativ neuen digitalen Format, Postings der Kommentarforen, charakterisieren, kann Folgendes festgehalten werden: Der Tabelle 1 sind Prozentsätze einzelner Formen der Intertextualität nach

8 Janich (2018: 190) diskutiert, man kann solche Beispiele entweder zu „Anspielungen auf die Struktur” oder aber zu ,inhaltlichen Anspielungen” zählen.

9 Diese Redewendung hat folgende übertragene Bedeutung „Daraus wird nichts.” oder „Das hat keinen Sinn." (Redensarten-Index). 
Referenztexten zu entnehmen, die anhand einer einfachen Statistik berechnet wurden. ${ }^{10}$ Die Intertextualität in Postings erfolgt formal vorwiegend durch die Übernahme von zentralen lexikalischen Elementen aus dem Zeitungsartikel (36 \%) oder aus den Referenzpostings $(37 \%)$, indem auf argumentative Gehalte aus dem Referenztext, die in Phänotexten argumentativ ergänzt oder kritisch kommentiert werden, angeknüpft wird. Bei Posting-Posting-Bezugnahmen werden in Phänopostings zudem relativ häufig (15\%) längere markierte Zitate (Sätze oder Absätze) aus Referenzpostings vorangestellt und/ oder vom Text abgesetzt übernommen, um widersprochen, kritisch kommentiert oder argumentativ ergänzt zu werden. Dadurch wird der Lesefluss unterstützt, denn dem Leser wird in der Flut unterschiedlicher Postings explizit gemacht, welche Textstelle aus welchem Posting nun kommentiert wird. Markiert zitierte Textstelle aus dem Zeitungsartikel sind hingegen viel seltener vorzufinden (4\%), was damit zusammenhängt, dass alle Leser den Ausgangstext nicht aber unbedingt alle vorangehenden Postings gelesen haben. In Postings wird zudem relativ häufig (20\%) auf satzwertige Phraseologismen wie Sprichwörter oder weitere Texte wie die Bologna-Erklärung verwiesen. Während satzwertige Phraseologismen an exponierten Stellen im Text unmarkiert zitiert werden (textstrukturierende Funktion), um das Thema des Postings interessant einzuführen, den Inhalt pointiert zu resümieren oder Argumente zu stützten, übernehmen Zitate aus weiteren Texten meist die argumentationsstützende Funktion. Einen Sonderfall stellen zitierte URLs dar (3 \%), die entweder durch das Deiktikon hier angekündigt werden oder das Deiktikon hier wird selbst verlinkt. Auf diese Weise wird ein Teil der Informationen, die beim Interesse nachgelesen werden können, ausgelagert und der Postingtext entlastet. Links tragen wesentlich zur Bedeutungserweiterung eines Postings bei und erfüllen entweder die argumentationsstützende oder veranschaulichende Funktion.

Tab. 1: Prozentsätze von Formen der Intertextualität nach Referenztext

\begin{tabular}{|c|c|c|c|c|c|c|}
\hline & \multicolumn{2}{|l|}{ Zitat } & \multirow{2}{*}{$\begin{array}{l}\text { Übernahme } \\
\text { von synt. } \\
\text { Strukturen } \\
\text { bei lex. Sub- } \\
\text { stitution }\end{array}$} & \multirow{2}{*}{$\begin{array}{l}\text { Verwendung } \\
\text { lex. Elemen- } \\
\text { ten. bei struk- } \\
\text { tureller Modifi- } \\
\text { kation }\end{array}$} & \multirow{2}{*}{$\begin{array}{l}\text { Übernahme } \\
\text { von lex. } \\
\text { Elementen./ } \\
\text { Synonymen }\end{array}$} & \multirow{2}{*}{$\begin{array}{l}\text { Inhaltliche } \\
\text { Anspie- } \\
\text { lung }\end{array}$} \\
\hline & $\begin{array}{l}\text { mar- } \\
\text { kiert }\end{array}$ & $\begin{array}{l}\text { unmar- } \\
\text { kiert }\end{array}$ & & & & \\
\hline Zeitungsartikel & \multicolumn{2}{|l|}{$4 \%$} & / & / & $36 \%$ & $8 \%$ \\
\hline & $4 \%$ & / & & & & \\
\hline \multirow{2}{*}{$\begin{array}{l}\text { Posting als } \\
\text { Referenztext }\end{array}$} & \multicolumn{2}{|c|}{$15 \%$} & $4 \%$ & $1 \%$ & $37 \%$ & $7 \%$ \\
\hline & $14 \%$ & $1 \%$ & & & & \\
\hline \multirow{2}{*}{$\begin{array}{l}\text { weitere Texte als } \\
\text { Referenztexte }\end{array}$} & \multicolumn{2}{|c|}{$20 \%$} & $1 \%$ & / & 1 & $1 \%$ \\
\hline & $6 \%$ & $14 \%$ & & & & \\
\hline
\end{tabular}

10 Für jedes Posting wird gezählt, welche der Formen vorkommen bzw. nicht vorkommen und nicht wie oft eine Form vorkommt. Wenn beispielsweise in einem Posting zwei markierte Zitate vorhanden sind, wird nur eins gezählt; kommen in einem Posting hingegen ein markiertes und ein unmarkiertes Zitat vor, so wird jede Form gezählt. 


\section{ZUSAMMENFÜHRUNG UND AUSBLICK}

Anhand der exemplarischen Analyse von 119 Postings aus drei Kommentarforen von Zeit-Online konnte in Anlehnung an Janischs formale Typologisierung eine eigene Typologie von intertextuellen Bezugnahmen unter Berücksichtigung technisch-medialer Charakteristika erarbeitet werden. Hierbei wurden nicht nur Bezüge auf Textelemente, sondern auch auf auditive und visuelle Elemente multimodaler Kommunikate berücksichtigt. Aufgrund medialer Prägung und veränderter textueller Praktik von Postings (Dialogizität, Interaktion u. a.) ist es notwendig, das komplexe und dichte Netz an intertextuellen Bezügen nach unterschiedlichen Referenztexttypen (Zeitungsartikel, Postings, andere Texte) getrennt zu beschreiben, um diese medial geprägte neue Qualität intertextueller Beziehungen zu erfassen. Obwohl in Postings etablierte intertextuelle Verweispraktiken wie die Übernahmen von zentralen lexikalischen Einheiten ohne Orientierung an syntaktischer Struktur sowie inhaltliche Anspielungen ohne Übernahme von lexikalischen Elementen und Strukturen dominieren, konnten auch einige Änderungen bzw. neue intertextuelle Verweispraktiken festgestellt werden: So werden in Postings relativ häufig längere Referenztextstellen zitiert, mit denen explizit markiert wird, welche (argumentative) Gehalte der Referenzpostings in der Flut aller Postings nun ergänzt, kommentiert oder kritisiert werden. Da längere Zitate markiert und vorangestellt in Phänopostings platziert sind, beeinflussen sie die Struktur der Phänopostings, was den Intertextualitätsgrad erhöht. Manchmal wird auf das Referenzposting explizit in der Überschrift durch Re: verwiesen, was an die E-Mail-Kommunikation erinnert. Technisch-medial neu sind intertextuelle Verweise auf beliebige Kommunikate durch Verlinkung, die kataphorisch oft durch das Deiktikon hier eingeleitet werden oder das Deiktikon selbst wird verlinkt, um den Postingtext zu entlasten und zum Weiterlesen zu animieren. Zudem werden intertextuelle Bezüge durch das hohe Interaktionspotenzial bzw. die Dialogizität beeinflusst: Einerseits ließen sich Verweise auf UserInnen (Zitieren von Usernamen) feststellen, um konkreten Postings dieser UserInnen zu kommentieren oder kritisieren; andererseits liegen in Postingswechseln Gesprächssequenzen vor, die hier als inhaltliche Anspielungen analysiert wurden. Diese reichen von scherzhaft-ironischen (Posting 1: Vergessen Sie es ... Posting 2: Ich vergesse nichts ;-)) bis unhöflich beleidigenden (Posting 1: Können Sie lesen? Posting 2: Ja, kann ich.). Unhöfliches sprachliches Verhalten oder auch falsche Rechtschreibung u. a. wird in Postings metasprachlich explizit kommentiert, indem formal meist auf Zitate zurückgegriffen wird. Schließlich überrascht es, dass trotz technisch-medialer Möglichkeiten, multicodale Texte einfach zu rekontextualisieren, in den untersuchten Kommentarforen keine audio-visuellen Referenzkommunikate und nur selten Postings mit audio-visuellen Bestandteilen gefunden werden konnten.

In Zukunft wären Postings zum einen in einem intermedialen Vergleich mit den Leserbriefen zu kontrastieren; zum anderen wäre korpusbasiert und sprachübergreifend zu untersuchen, ob auch bei Postings die bei den Leserbriefen vorkommende kulturelle Prägung festzustellen ist. 


\section{Primärliteratur}

KLIMKEIT, Lena (2015) „Bologna: Geschichte einer Enttäuschung.“ https://www. zeit.de/studium/hochschule/2015-04/bachelor-studie-unternehmen-unzufrieden [10.02.2019].

Studentenproteste: Uni-Rektoren geben Ländern die Schuld an Bachelor-Chaos. (2009) https://www.zeit.de/studium/hochschule/2009-11/studentenproteste-hrk-leipzig [10.02.2019].

TÖCHTERLE, Karlheinz(2014) ECTS: Punkt um Punkt. https://www.zeit.de/2014/48/ ects-studienfortschritt-universitaeten-europa [10.02.2019].

\section{Sekundärliteratur}

ADAMZIK, Kirsten (2004) Textlinguistik. Eine einführende Darstellung. Tübingen: Niemeyer.

ANDROUTSOPOULOS, Jannis (2016) „Mediatisierte Praktiken: Zur Rekontextualisierung von Anschlusskommunikation in den sozialen Medien." In: A. Deppermann/H. Feilke/A. Linke (Hrsg.), Sprachliche und kommunikative Praktiken. Berlin/Boston: de Gruyter, 337-367.

BERGHOFER, Simon (2009) Dialogizität und [Inter] Textualität im Internet: Zur kommunikativen Textgenese von literarischen Blogs. Magisterarbeit. Freie Universität Berlin. Berlin.

BRECKLE, Margit/Mona ENELL-NILSSON (2015) „Methodische Herausforderungen der Analyse intertextueller Bezüge - am Beispiel von Editorials in Kundenzeitschriften." In: D. Rellstab/N. Siponkoski (Hrsg.), Rajojen dynamiikkaa, Gränsernas dynamik, Borders under Negotiation, Grenzen und ihre Dynamik. VAKKI-symposiumi XXXV 12.-13.2.2015. Vaasa: VAKKI Publications, 86-95.

BRINKER, Klaus/Sven F. SAGER (2006) Linguistische Gesprächsanalyse. Eine Einführung. 4., durchges. und erg. Aufl. Berlin: Erich Schmidt. BROICH, Ulrich/Manfred PFISTER (Hrsg.) (1985) Intertextualität. Formen, Funktionen, anglistische Fallstudien. Tübingen: Niemeyer. DEPPERMANN, Arnulf/Helmuth FEILKE/ Angelika LINKE (2016), ,Sprachliche und kommunikative Praktiken: Eine Annäherung aus linguistischer Sicht." In: A. Deppermann/ H. Feilke/A. Linke (Hrsg.), Sprachliche und kommunikative Praktiken. Berlin/Boston: de Gruyter, 1-23.

ECKKRAMMER, Eva Martha/Gabriele KNAUER (2015) „Kommunikative Handlungsmuster im Wandel: theoretische und methodische Bausteine ausgelotet anhand der Textsorte Leserbrief.“ In: N. Rentel/T. Schröder/R. Schröpf (Hrsg.), 249-281.

FANDRYCH, Christian/Maria THURMAIR (2011) Textsorten im Deutschen. Linguistische Analysen aus sprachdidaktischer Sicht. Tübingen: Stauffenburg.

GRAY, Jonathan (2006) Watching with the Simpsons: Television, parody, and intertextuality. New York: Routledge.

GROSSE, Sybille (2015) „Kommentare politischer Blogs - Interaktion zwischen Konvention und sprachlicher Freiheit. Blog Generación $Y$. “ In: N. Rentel/T. Schröder/R. Schröpf (Hrsg.), 35-65. 
HEIBACH, Christiane (2003) Literatur im elektronischen Raum. Frankfurt a. Main: Suhrkamp.

HOLTHUIS, Susanne (1993) Intertextualität. Aspekte einer rezeptionsorientierten Konzeption. Tübingen: Stauffenburg.

JANICH, Nina (2019) „Intertextualität und Text(sorten)vernetzung.“ In: N. Janich (Hrsg.), Textlinguistik: 15 Einführungen. 2. Aufl. Tübingen. Narr, 177-196.

KLUG, Nina-Maria (2018) „Wenn schlüsseltexte Bilder sind. Aspekte von Intertextualität in Presse und öffentlichem Raum.“ In: S. Pappert/S. Michel (Hrsg.), Multimodale Kommunikation in öffentlichen Räumen. Texte und Textsorten zwischen Tradition und Innovation. Stuttgart: ibidem-Verlag, 109-131.

LÜGER, Heinz-Helmut (1999) Satzwertige Phraseologismen. Eine pragmalinguistische Untersuchung. Wien: Praesens.

MARX, Konstanze/Georg WEIDACHER (2014) Internetlinguistik: Ein Lehr- und Arbeitsbuch. Tübingen: Narr Francke Attempto.

OPIŁOWSKI, Roman (2006) Intertextualität in der Werbung der Printmedien. Eine Werbestrategie in linguistisch-semiotischer Forschungsperspektive. Frankfurt a. Main [etc.].: Peter Lang.

PFISTER, Manfred (1985) „Konzepte der Intertextualität.” In: U. Broich/m. Pfister (Hrsg.), 1-30.

RENTEL, Nadine/Tilman SCHRÖDER/Ramona SCHRÖPF (Hrsg.) (2015) Kommunikative Handlungsmuster im Wandel? ¿Convenciones comunicativas en proceso de transformación? Chats, Foren und Dienste des Web 2.0 im deutsch-spanischen Vergleich. Frankfurt a. Main: Peter Lang. SPIEß, Constanze (2018) „Stancetakingund Positionierungsaktivitäten im Öffentlichen Metasprachdiskurs über Jugendliche Sprechweisen: Eine Analyse von User*innen-Kommentaren im Web.” In: A. Ziegler (Hrsg.), Jugendsprachen/Youth Languages: Aktuelle Perspektiven internationaler Forschung/Current Perspectives of International Research. Berlin/Boston: de Gruyter, 147-188.

SPITZMÜLLER, Jürgen/Ingo H. WARNKE (2011) Diskurslinguistik. Eine Einführung in Theorien und Methoden der transtextuellen Sprachanalyse. Berlin/Boston: de Gruyter.

WEIDACHER, Georg (2017) „Textsorten als dynamische, medial geprägte kommunikative Praxen. Diskussion eines medienlinguistischen Textsortenbegriffs anhand der exemplarischen Analyse von Postings in Kommentar- und Diskussionsforen." In: Z. Bilut-Homplewicz/A. Hanus/A. Mac (Hrsg.), Medienlinguistik und interdisziplinäre Forschung I: Textsortenfragen im medialen Umfeld. Frankfurt a. Main: Peter Lang, 151-170. ZIMMERMANN, Tobias (2017) Digitale Diskussionen. Über politische Partizipation mittels Online-Leserkommentaren. Bielefeld: Transcript.

\section{Internetquellen}

NATH, Dörthe „68er-Revolte - „Der NS-Muff ist vertrieben“. Interview mit Detlev Albers. Süddeutsche Zeitung. 7. November 2007 [03.02.2019].

Redenarten-Index. https://www.redensarten-index.de/suche.php [11.2.2019]. 


\section{Zusammenfassung \\ ZUR INTERTEXTUALITÄT IN POSTINGS: EINE EXEMPLARISCHE ANALYSE DEUTSCHSPRACHIGER POSTINGS IN KOMMENTARFOREN ZU ONLINE-ZEITUNGSARTIKELN ZUM BOLOGNA-PROZESSABSTRACT}

Im Beitrag werden vielfältige intertextuelle Bezüge in Postings der Kommentarforen, die für diese junge kommunikative Praktik konstitutiv sind, differenziert beschrieben. Postings nehmen als reaktive kommunikative Praktiken nicht nur auf die Online-Zeitungsartikel, sondern auch auf Postings anderer UserInnen sowie auf weitere Texte intertextuell Bezug. Aufgrund medialer Prägung und veränderter textueller Praktik von Postings (Dialogizität, Interaktion u. a.) ist es notwendig, intertextuelle Bezüge nach unterschiedlichen Referenztexten getrennt zu beschreiben, um diese medial geprägte neue Qualität intertextueller Beziehungen zu erfassen. Anhand der Analyse von 119 Postings aus drei Kommentarforen von Zeit-Online konnte eine eigene Typologie von intertextuellen Bezugnahmen unter Berücksichtigung technisch-medialer Charakteristika erarbeitet werden. Hierbei wurden nicht nur Bezüge auf Textelemente, sondern auch auf auditive und visuelle Elemente multimodaler Kommunikate berücksichtigt. Obwohl in Postings etablierte intertextuelle Verweise wie die Übernahmen von zentralen lexikalischen Einheiten sowie inhaltliche Anspielungen dominieren, konnten auch einige Änderungen (längere vorangestellte Zitate) bzw. neue intertextuelle Bezugnahmen (Verlinkungen, auf mehrere Postings verteile Gesprächssequenzen u. a.) festgestellt werden.

Schlüsselwörter: Intertextualität, Postings der Kommentarforen, kommunikative Praktik, computervermittelte Kommunikation, Dialogizität

\section{Abstract \\ INTERTEXTUALITY IN COMMENTS ON ONLINE ARTICLES: AN ANALYSIS OF COMMENTS ON GERMAN ONLINE ARTICLES ABOUT THE BOLOGNA PROCESS}

The paper focuses on different forms of intertextuality in comments on online articles which are characteristic of this communicative practice. Comments can refer either to online articles or to comments of other users as well as to other verbal and non-verbal texts. Due to media-related constraints and the modified textual practices of computermediated communication (dialogicity, interaction etc.), intertextual relations have to be described for each different type of reference text separately. Only in this way can we adequately describe the new quality of intertextuality influenced by media. Based on the analysis of 119 comments on three different online articles referring to the Bologna reform from the German online newspaper Zeit-Online, a typology of different forms of intertextual relations was proposed, taking into consideration both technical and media aspects. Intertextual references on textual, auditive and visual elements were all 
considered. Although established forms of intertextuality were found to be the most common in online comments (e.g. reproduction of key lexical elements and allusions to the content), some changes of intertextual references (longer citations at the beginning of comments) or new forms (links, speech sequences etc.) were identified.

Key words: intertextuality, comments, communicative practice, computer-mediated communication, interaction

\section{Povzetek}

\section{MEDBESEDILNOST V SPLETNIH KOMENTARJIH: EKSEMPLARIČNA ANALIZA NEMŠKIH SPLETNIH KOMENTARJEV K ČLANKOM O BOLONJSKI REFORMI}

Prispevek se osredinja na medbesedilnost v spletnih komentarjih, ki je značilna za to mlado komunikativno prakso spletno posredovane komunikacije. Spletni komentarji se namreč lahko medbesedilno nanašajo na spletne članke, komentarje drugih uporabnikov ter druga besedila. Zaradi značilnosti računalniško posredovane komunikacije in posledično spremenjenega ubesedilovanja $\mathrm{v}$ spletnih komentarjih (dialoškost, interaktivnost itd.) je pomembno medbesedilnost analizirati in opisati po posameznih vrstah predlog, da bi lahko tako ustrezno opisali nove oblike medbesedilnosti. Na osnovi analize 119 spletnih komentarjev s treh spletnih forumov iz spletnega časopisa Zeit-Online smo izdelali tipologijo oblik medbesedilnosti ob upoštevanju tehničnih medijskih značilnosti. Vključili smo tako nanašanja na besedilne kot tudi na avditivne in vizualne elemente. Čeprav sta v spletnih komentarjih najpogostejši ustaljeni obliki medbesedilnosti privzemanje ključnih leksikalnih sestavin in vsebinsko nanašanje, so se pojavile tudi nekatere spremenjene (postavljanje daljših citatov na začetek spletnega komentarja) oz. nove oblike medbesedilnega nanašanja (citiranje linkov, govorne sekvence itd.).

Ključne besed: medbesedilnost, spletni komentarji, komunikativne praktike, računalniško posredovana komunikacija, dialoškost 